\title{
Fabrication and characterization of nuclear localization signal-conjugated glycol chitosan micelles for improving the nuclear delivery of doxorubicin
}

\author{
Jingmou $\mathrm{Yu}^{\prime}$ \\ Xin Xie \\ Meirong Zheng' \\ Ling $\mathrm{Yu}^{2}$ \\ Lei Zhang' \\ Jianguo Zhao' \\ Dengzhao Jiang' \\ Xiangxin Che \\ 'Key Laboratory of Systems Biology \\ Medicine of Jiangxi Province, College \\ of Basic Medical Science, Jiujiang \\ University, Jiujiang, ${ }^{2}$ Division of \\ Nursing, 2nd Affiliated Hospital, \\ Yichun University, Yichun, People's \\ Republic of China
}

\author{
This article was published in the following Dove Press journal: \\ International Journal of Nanomedicine \\ 20 September 2012 \\ Number of times this article has been viewed
}

Background: Supramolecular micelles as drug-delivery vehicles are generally unable to enter the nucleus of nondividing cells. In the work reported here, nuclear localization signal (NLS)modified polymeric micelles were studied with the aim of improving nuclear drug delivery. Methods: In this research, cholesterol-modified glycol chitosan (CHGC) was synthesized. NLSconjugated CHGC (NCHGC) was synthesized and characterized using proton nuclear magnetic resonance spectroscopy, dynamic light scattering, and fluorescence spectroscopy. Doxorubicin (DOX), an anticancer drug with an intracellular site of action in the nucleus, was chosen as a model drug. DOX-loaded micelles were prepared by an emulsion/solvent evaporation method. The cellular uptake of different DOX formulations was analyzed by flow cytometry and confocal laser scanning microscopy. The cytotoxicity of blank micelles, free DOX, and DOX-loaded micelles in vitro was investigated by 3-(4,5-dimethylthiazol-2-yl)-2,5-diphenyltetrazolium bromide (MTT) assay in HeLa and HepG2 cells.

Results: The degree of substitution was 5.9 cholesterol and 3.8 NLS groups per 100 sugar residues of the NCHGC conjugate. The critical aggregation concentration of the NCHGC micelles in aqueous solution was $0.0209 \mathrm{mg} / \mathrm{mL}$. The DOX-loaded NCHGC (DNCHGC) micelles were observed as being almost spherical in shape under transmission electron microscopy, and the size was determined as $248 \mathrm{~nm}$ by dynamic light scattering. The DOX-loading content of the DNCHGC micelles was $10.1 \%$. The DOX-loaded micelles showed slow drug-release behavior within 72 hours in vitro. The DNCHGC micelles exhibited greater cellular uptake and higher amounts of DOX in the nuclei of HeLa cells than free DOX and DOX-loaded CHGC (DCHGC) micelles. The half maximal inhibitory concentration $\left(\mathrm{IC}_{50}\right)$ values of free DOX, DCHGC, and DNCHGC micelles against HepG2 cells were 4.063, 0.591, and $0.171 \mu \mathrm{g} / \mathrm{mL}$, respectively. Moreover, the $\mathrm{IC}_{50}$ values of free DOX $(3.210 \mu \mathrm{g} / \mathrm{mL})$ and the DCHGC micelles $(1.413 \mu \mathrm{g} / \mathrm{mL})$ against HeLa cells were nearly 6.96- and 3.07-fold $(P<0.01)$, respectively, higher than the $\mathrm{IC}_{50}$ value of the DNCHGC micelles $(0.461 \mu \mathrm{g} / \mathrm{mL})$.

Conclusion: The results of this study suggest that novel NCHGC micelles could be a potential carrier for nucleus-targeting delivery.

Keywords: polymeric micelles, drug delivery, nucleus-targeting delivery

Xiangxin Che

College of Basic Medical Science, Jiujiang University, No 17 Lufeng Road, Jiujiang, Jiangxi Province 332000,

People's Republic of China

Tel +867928577050

Fax +867928577050

Emailyjm 1016@hotmail.com;

chexiangxin@hotmail.com

\section{Introduction}

Polymeric micelles as potential carriers for drug delivery have triggered considerable interest in recent years. ${ }^{1-5}$ It is well-known that amphiphilic copolymers are able to self-assemble into polymeric micelles in aqueous solution and form a unique core-shell structure. The hydrophobic core can serve as a reservoir for poorly water-soluble drugs, 
while the modification of the hydrophilic shell can affect pharmacokinetic behavior. The size of polymeric micelles is approximately nanoscale, which not only makes them accumulate selectively in tumor tissues due to an enhanced permeability and retention effect, but also offers a good opportunity for efficient intracellular anticancer drug delivery. ${ }^{6,7}$ In particular, during the preparation of micellebased drug-delivery systems, much attention has been paid to biodegradable and nontoxic polymeric amphiphiles. ${ }^{8,9}$

Chitosan, a natural aminopolysaccharide obtained by the deacetylation of chitin, has been used as a drug-delivery vehicle because of its favorable biological properties such as biocompatibility, biodegradability, positive charge, low immunogenicity and biological activity. ${ }^{10}$ However, its application has been restricted due to its poor solubility in neutral or basic aqueous solutions. ${ }^{11}$ Recently, many watersoluble chitosan derivatives have been prepared. Glycol chitosan, a novel chitosan derivative, is soluble in water at all $\mathrm{pH}$ values. Hydrophobically modified glycol chitosan derivatives can form stable self-aggregated nanoparticles in biological media solutions and be used as carriers for hydrophobic drugs and genes. ${ }^{12-15}$ In our previous studies, cholesterol-modified glycol chitosan (CHGC) conjugate was synthesized and formed aggregated micelles in an aqueous medium. ${ }^{16}$ In addition, the doxorubicin (DOX)-loaded CHGC nanoparticles exhibited prolonged circulation in the blood and showed higher antitumor efficacy against S180 tumorbearing mice through an enhanced permeability and retention effect than free DOX. ${ }^{17}$

DOX is an anthracycline antibiotic drug that has a broad antitumor spectrum in the clinical field. Its action takes place inside the nucleus of cancer cells, where DOX induces apoptosis by binding to DNA and reacting with a variety of biomolecules. ${ }^{18}$ Unfortunately, it interacts with both healthy and cancerous tissues, giving rise to dose-limiting adverse effects such as cardiotoxicity and myelosuppression. To maximize the efficacy of DOX in tumor tissues and minimize the toxicity to normal tissue, nanocarriers such as liposomes, microemulsions and micelles have been studied. ${ }^{19,20}$ Notably, as we know, large foreign molecular substances, including proteins, genes, and polymeric micelles, are mostly unable to enter the nucleus of nondividing cells, in which the nuclear membrane does not disappear upon cell division, since nuclear transfer is strictly controlled by precise machinery. ${ }^{21}$ The nuclear envelope contains specialized channels called the 'nuclear pore complex' (NPC), which allow passive diffusion of ions and small molecules $(<40 \mathrm{kDa})$ through aqueous channels. ${ }^{22}$ Molecules of large size ( $>25 \mathrm{~nm}$ in diameter) are transported across the NPC by specific nuclear import and export systems. ${ }^{21}$ Therefore, efficient nuclear drug delivery may be an effective approach to increase the therapeutic efficacy of DOX. The nucleus is most commonly targeted through the conjugation of a nuclear localization signal (NLS) to the surface of nanoparticles. ${ }^{23-25}$ Classical NLS consists of either one or two stretches of the basic amino acids arginine and lysine. It has been reported that classical NLSs on the macromolecule or "cargo" to be transported are recognized by importin $\alpha / \beta$ heterodimers and that the cargo-receptor complex crosses the NPC. ${ }^{26}$

The primary objective of the work here presented was to construct novel DOX-loaded polymeric micelles for enhanced nuclear delivery. A NLS (Ac-CGYGPKKKRKVGG)modified CHGC conjugate was synthesized and characterized. DOX-loaded NLS-conjugated CHGC (DNCHGC) micelles were prepared by an emulsion/solvent evaporation method. The physicochemical properties of the DNCHGC micelles were investigated and the intracellular delivery of DOX formulations was also studied. Moreover, analysis of the cytotoxicity of DNCHGC micelles was carried out in HeLa and HepG2 cancer cells.

\section{Materials and methods Materials}

Glycol chitosan $\left(\mathrm{M}_{\mathrm{w}}=4.3 \times 10^{5}, 75.2 \%\right.$ of degree of deacetylation determined by titration) and potassium poly(vinyl sulfate) were purchased from Wako Pure Chemical Industries, Ltd (Osaka, Japan). Cholesterol, succinic anhydride, cellulase, $\mathrm{N}$-succinimidyl 4-(maleimidomethyl) cyclohexane carboxylate (SMCC), 5,5'-dithiobis(2nitrobenzoic acid), and 3-(4, 5-dimethyl-thiazol-2-yl)-2, 5-diphenyl-tetrazolium bromide (MTT) were purchased from Sigma-Aldrich (St Louis, MO). 1-Ethyl-3-(3dimethylaminopropyl)-carbodiimide hydrochloride (EDC) was obtained from Acros Organics (Fair Lawn, NJ) and used without further purification. Pyrene, purchased from Acros Organics, was recrystallized twice from anhydrous ethanol before use. Doxorubicin hydrochloride was purchased from the Beijing Huafeng United Technology Co, Ltd (Beijing, China). Roswell Park Memorial Institute (RPMI) 1640 medium and trypsin-ethylenediaminetetraacetic acid (EDTA) were supplied by the Jinuo Biotechnology Co (Hangzhou, China). Fetal bovine serum (FBS) was purchased from Sijiqing Biologic Co, Ltd (Zhejiang, China). The Ac-CGYGPKKKRKVGG peptide $\left(\mathrm{M}_{\mathrm{w}}=1420 \mathrm{Da}\right)$ was synthesized by APeptide Co, Ltd (Shanghai, China). Hoechst 33342 was obtained from the Beyotime Institute of 
Biotechnology (Haimen, China). All other chemicals were of analytical grade and used as received.

\section{Degradation of glycol chitosan}

Due to the high viscosity of commercially supplied glycol chitosan, degraded glycol chitosan was produced by enzymatic degradation and membrane technology. ${ }^{27,28}$ First, glycol chitosan $(6 \mathrm{~g})$ was dissolved in $400 \mathrm{~mL}$ of distilled water overnight. Then $11 \mathrm{~mL}$ of acetic acid and cellulase $(0.12 \mathrm{~g})$ were added under magnetic stirring. The temperature of the solution was raised to $50^{\circ} \mathrm{C}$. After 8 hours, this reaction mixture was exhaustively dialyzed (molecular weight cutoff [MWCO] $3.5 \mathrm{kDa}$ ) against distilled water for 24 hours. Following this, the dialyzed solution was ultrafiltered using various MWCO ultrafiltration membranes (Amicon Stirred Cell 8400, Millipore, Billerica, MA). Thereafter, the degraded glycol chitosan was obtained by lyophilization. The molecular weight of the glycol chitosan was analyzed by gel permeation chromatography with a TSK-gel column (G3000SW, $7.5 \mathrm{~mm}$ ID $\times 30 \mathrm{~cm}$, Tosoh Corporation, Tokyo, Japan) at $25^{\circ} \mathrm{C}$. The mobile phase was acetate buffer solution ( $\mathrm{pH}$ 6.0) with a flow rate of $0.8 \mathrm{~mL} / \mathrm{min}$. The average molecular weight of the degraded glycol chitosan was determined to be $28 \mathrm{kDa}$ in the present study.

\section{Synthesis of the CHGC conjugate}

The CHGC conjugate was synthesized using a previously reported method with little modification. ${ }^{16}$ Briefly, cholesterol $(4.0 \mathrm{~g})$ was dissolved in $16 \mathrm{~mL}$ of pyridine then succinic anhydride (2.88 g) was added. After 24 hours, the mixture was precipitated in water. The obtained precipitate was filtered then dissolved in ether before being filtered again to remove the byproducts. The obtained solution was dried under reduced pressure. The white powder of cholesterol hemisuccinate (CHS; $88 \%$ yield) was obtained by recrystallization in acetone. Glycol chitosan $(500 \mathrm{mg}$ ) was dissolved in $30 \mathrm{~mL}$ of distilled water and diluted with $106 \mathrm{~mL}$ of ethanol. Then EDC (61.4 mg, $0.32 \mathrm{mmol})$ and CHS (77.9 mg, $0.16 \mathrm{mmol}$ ) were slowly added. After the reaction mixture was stirred for 72 hours at room temperature, it was sequentially dialyzed (MWCO $3.5 \mathrm{kDa}$ ) against an excess amount of ethanol/distilled water solution $(88: 12, \mathrm{v} / \mathrm{v})$ and distilled water, each for 2 days with several exchanges. The dialyzed solution was freeze-dried to obtain CHGC.

The chemical structure of the CHGC conjugate was determined by proton nuclear magnetic resonance ( ${ }^{1} \mathrm{H}$ NMR) spectroscopy (Avance DMX-500 spectrometer, Bruker Optics, Billerica MA). Heavy water $\left(\mathrm{D}_{2} \mathrm{O}\right)$ was used as the solvent. The degree of substitution (DS), defined as the number of cholesterol groups per 100 sugar residues of glycol chitosan, was determined by a colloidal titration method..$^{29} \mathrm{In}$ brief, CHGC ( $5 \mathrm{mg}$ ) was dissolved in an aqueous $2 \%$ acetic acid solution $(5 \mathrm{~mL})$ and placed in a beaker on a magnetic stirrer. After adding $100 \mu \mathrm{l}$ of the indicator, $0.1 \%(\mathrm{w} / \mathrm{v})$ toluidine blue, the CHGC suspension was slowly titrated with $2.5 \mathrm{mM}$ potassium poly(vinyl sulfate) solution until the opaque solution suddenly became clear.

\section{Synthesis of the NLS-conjugated CHGC (NCHGC) conjugate}

CHGC (100 mg) was dissolved in $40 \mathrm{~mL}$ of distilled water ( $\mathrm{pH}$ 8.0) then sonicated 40 times using a probe-type sonifier (JY92-II, Ningbo Scientz Bio-tech Co, Ltd, Ningbo, China) at $200 \mathrm{~W}$ in an ice bath. Then SMCC $(7.2 \mathrm{mg}, 0.0215 \mathrm{mmol})$ dissolved in $0.5 \mathrm{~mL}$ of dimethyl sulfoxide (DMSO) was added. The mixture was incubated at room temperature for 2 hours. After adjusting the $\mathrm{pH}$ of the mixture to 5.5, synthesized NLS peptides $(45 \mathrm{mg})$ were added. The mixture was stirred at room temperature for another 4 hours. The unconjugated NLS peptides were removed using the centrifugal-ultrafiltration method described by Du et al. ${ }^{30}$ The mixture was placed into centrifugal-ultrafiltration tubes (Amicon Ultra-15, MWCO 3000 , Millipore) and centrifuged at $5000 \mathrm{rpm}$ at $4^{\circ} \mathrm{C}$ for 40 minutes. This step was repeated twice with the addition of distilled water until free unreacted NLS was not detected. The chemical structure of the $\mathrm{NCHGC}$ conjugate was investigated by ${ }^{1} \mathrm{H}$ NMR. $\mathrm{D}_{2} \mathrm{O}$ was used as the solvent. The amount of NLS peptide conjugated to the micelles was determined by Ellman's assay protocol, which detects the terminal cysteine of NLS. ${ }^{31}$ The unreacted NLS, or initially used NLS peptides, were dissolved in Ellman's working solution (phosphatebuffered saline [PBS], $\mathrm{pH}=8.0$, containing $1 \mathrm{mM}$ EDTA) and Ellman's reagent solution was immediately added. Then the mixture was incubated for 15 minutes at room temperature. After the solution was filtered, the absorbance at $412 \mathrm{~nm}$ was measured and the NLS peptide levels were quantitated using a standard curve prepared with cysteine as the active reagent. The amount of unattached NLS was determined then this was subtracted from the total amount of NLS initially fed into the reaction to obtain the amount of NLS conjugated to the NCHGC micelles.

The particle size and zeta potential were analyzed using a 90Plus Particle Size Analyzer (Brookhaven Instruments Corporation, Holtsville, NY). The micelles were dispersed in deionized water. The particle size analysis was carried out at a scattering angle of $90^{\circ}$ and at a temperature of $25^{\circ} \mathrm{C}$. 
The zeta potential values were measured at $25^{\circ} \mathrm{C}$ and at the default parameters of dielectric constant, refractive index, and viscosity of water. All measurements were performed in triplicate.

\section{Determination of critical aggregation concentration (CAC)}

NCHGC samples for the fluorescence investigation were prepared by probe sonication as described earlier. A known amount of pyrene in acetone was added into each of $10-\mathrm{mL}$ vials, and acetone was evaporated at $40^{\circ} \mathrm{C}$. A total of $10 \mathrm{~mL}$ of various concentrations of NCHGC conjugate were added to each vial and heated at $50^{\circ} \mathrm{C}$ for 10 hours to equilibrate the pyrene and micelles. The vials were then left undisturbed to cool overnight at room temperature. The final concentration of pyrene was $6.0 \times 10^{-7} \mathrm{M}$. Steady-state fluorescent spectra were measured using a fluorescence spectrophotometer (Hitachi F-4500, Tokyo, Japan) with a slit width of $2.5 \mathrm{~nm}$. The excitation and emission wavelengths were set at 339 and $390 \mathrm{~nm}$, respectively.

\section{Preparation and characterization of DOX-loaded micelles}

DOX-loaded micelles were prepared according to the emulsion/solvent evaporation method. ${ }^{25,32}$ The DOX base was obtained by reacting doxorubicin hydrochloride with the equivalent of $3 \mathrm{~mol}$ of triethylamine (TEA) in chloroform overnight then used for the preparation of the drug-loaded micelles. Copolymer samples (30 mg, CHGC or NCHGC) were dissolved in distilled water $(60 \mathrm{~mL})$, followed by probe sonication as described earlier. Then a $3 \mathrm{~mL}$ chloroform solution of DOX (4.5 mg) was added to the aqueous phase under vigorous stirring to form the oilin-water emulsion. The mixture was stirred under reduced pressure, allowing the slow evaporation of chloroform and micelle formation. The aqueous suspension was eluted through a fine column $(1.6 \mathrm{~cm} \times 35 \mathrm{~cm}$, Shanghai Troody Analytical Instrument Co, Ltd, Shanghai, China) loaded with Sephadex ${ }^{\circledR}$ G25 to separate the DOX-encapsulated micelles from the nonencapsulated DOX. The drug-loaded micelles were lyophilized for future use.

The morphology of the drug-loaded micelles was observed using a JEM 1230 transmission electron microscope (JEOL, Tokyo, Japan) operating at an acceleration voltage of $80 \mathrm{kV}$. A drop of sample solution was placed onto a 300mesh copper grid coated with carbon and extra solution was blotted with filter paper before being air dried. Negative staining was performed using a droplet of $2 \mathrm{wt} \%$ phospho- tungstic acid solution. The particle size and zeta potential of these nanoparticles were measured at $25^{\circ} \mathrm{C}$ by dynamic light scattering (DLS; 90Plus Particle Size Analyzer) as already described.

The drug loading content (LC) and entrapment efficiency (EE) were quantified by determining absorbance at $481 \mathrm{~nm}$ with an ultraviolet (UV)-visible spectrophotometer (Beijing Purkinje General Instrument Co, Ltd, Beijing, China). The freeze-dried samples were dispersed in aqueous solution and DMSO was added to dissociate the micelles. DOX solutions of various concentrations were prepared and the absorbance at $481 \mathrm{~nm}$ was measured to generate a calibration curve for LC calculations of the various micelles. The LC and EE were calculated by the following equations: LC $(\%)=$ the weight of DOX in micelles/total weight of micelles $\times 100$ and $\mathrm{EE}$ $(\%)=$ the weight of DOX in micelles/weight of DOX fed initially $\times 100$.

\section{In vitro DOX release}

In vitro release behavior of DOX from the drug-loaded micelles was studied using a dialysis method. ${ }^{17}$ Briefly, $1 \mathrm{~mL}$ of DOX-loaded micelles was sealed in a dialysis bag (MWCO $3.5 \mathrm{kDa}$ ), and incubated in $20 \mathrm{~mL}$ of PBS (pH 7.4) containing $10 \% \mathrm{FBS}$ at $37^{\circ} \mathrm{C}$ in an air-bath shaker at $100 \mathrm{rpm} .{ }^{33}$ At a predetermined time, all the media were replaced with the same amount of pre-warmed fresh media. The released drug was quantified using the F-4500 fluorescence spectrophotometer. Measurements were made at an excitation wavelength of $470 \mathrm{~nm}$ and emission wavelength of $585 \mathrm{~nm}$.

\section{Cell culture}

HeLa and HepG2 cells were obtained from the Institute of Biochemistry and Cell Biology of the Chinese Academy of Sciences (Shanghai, China). The cells were cultured in RPMI 1640 medium containing 10\% FBS and 1\% penicillinstreptomycin at $37^{\circ} \mathrm{C}$ in a humidified atmosphere containing $5 \% \mathrm{CO}_{2}$.

\section{Flow cytometry}

Flow cytometry was used to determine the uptake of DOX into the HeLa cells. ${ }^{34}$ The cells were plated in six-well plates at a density of $1 \times 10^{5}$ cells $/ \mathrm{mL}$ and cultured overnight at $37^{\circ} \mathrm{C}$ in $5 \% \mathrm{CO}_{2}$ to allow them to adhere at the bottom of the plates. After removing the cultured media, the cells were incubated with free DOX, DOX-loaded CHGC (DCHGC), or DNCHGC micelles (equivalent DOX concentration: $10 \mu \mathrm{g} / \mathrm{mL}$ ) in RPMI 1640 medium supplemented with $10 \%$ FBS. After 2 hours, the cells were washed three times with 
$1 \mathrm{~mL}$ of PBS (pH 7.4) then detached with trypsin-EDTA. This suspension was centrifuged at $1000 \mathrm{rpm}$ for 10 minutes. The supernatants were discarded and the cells were resuspended with PBS. The cells placed in the Falcon tubes were introduced into a flow cytometer (FACSCalibur, Becton, Dickinson and Company, Franklin Lakes, NJ) equipped with a $488 \mathrm{~nm}$ argon ion laser. The data presented are the mean fluorescent signals for 10,000 cells. All experiments were done in triplicate.

\section{Confocal microscope study}

For confocal microscopic observation, HeLa cells were seeded into $35 \mathrm{~mm}$ glass-bottomed cell culture dishes (Nest Biotechnology Co, Ltd, Wuxi, China) at a density of $1 \times 10^{5}$ cells $/ \mathrm{mL}$, and cultured for 48 hours..$^{35}$ Then free DOX, DNCHGC, or DCHGC micelles (equivalent DOX concentration: $5 \mu \mathrm{g} / \mathrm{mL}$ ) in RPMI 1640 medium supplemented with $10 \%$ FBS were added to each designated dish. After 2 hours incubation, all media were removed. The cells were washed three times with PBS, fixed in $4 \%$ paraformaldehyde for 10 minutes, and treated with Hoechst $33342(5 \mu \mathrm{g} / \mathrm{mL})$ for 20 minutes for staining the nuclei. After the cells were washed three times with PBS, they were imaged using a Zeiss LSM-510 confocal microscope (Jena, Germany) with identical settings for each confocal study. The intracellular localization of DOX was observed by confocal microscope at $488 \mathrm{~nm}$. A UV laser (405 nm excitation) was used to induce the blue fluorescence of Hoechst 33342.

\section{In vitro cytotoxicity}

The in vitro cytotoxicity of free DOX, blank micelles, and DOX-loaded micelles was investigated by MTT assay in HeLa and HepG2 cells. ${ }^{36}$ The cells were seeded at a density of $1 \times 10^{4}$ cells/well in 96-well plates (Costar, Corning, $\mathrm{NY}$ ) and allowed to grow for 24 hours. After removing the medium, $200 \mu \mathrm{L}$ of RPMI 1640 medium containing various concentrations of free DOX, CHGC, NCHGC, DCHGC, or DNCHGC micelles were added, and further incubated for 48 hours at $37^{\circ} \mathrm{C}$. At indicated time points, $30 \mu \mathrm{L}$ of MTT solution $(5 \mathrm{mg} / \mathrm{mL}$ in PBS) were added to each well. After a further 4 hours of incubation, the culture medium was removed and the formazan crystals in the cells were solubilized with $200 \mu \mathrm{L}$ of DMSO for 15 minutes. The UV absorbance at $570 \mathrm{~nm}$ was measured with a microplate reader (Multiskan MK3, Thermo Scientific, Hudson, OH). The sample-free wells were used as the control. Cytotoxicity was illustrated using the half maximal inhibitory concentration $\left(\mathrm{IC}_{50}\right)$. $\mathrm{IC}_{50}$ values were calculated using a sigmoidal dose-response model from GraphPad Prism (v 4.0; GraphPad Software Inc, La Jolla, CA). All experiments were performed in triplicate.

\section{Data analysis}

The statistical significance of the differences between groups was evaluated by one-way analysis of variance with a Bonferroni posthoc test. A $P$ value of $<0.05$ was considered significant.

\section{Results and discussion Characterization of CHGC micelles}

We prepared degraded glycol chitosan by an enzymatic degradation and ultrafiltration method. CHGC was synthesized by coupling the carboxyl groups of CHS with the amino groups of glycol chitosan in the presence of EDC (Figure 1). The incorporation of CHS into the glycol chitosan was confirmed by the characteristic peak of CHS in the ${ }^{1} \mathrm{H}$ NMR spectra (Figure 2). The proton assignment of glycol chitosan (Figure 2A) is as follows (ppm): $1.95\left(\mathrm{CH}_{3}\right.$, methyl protons of acetyl group of $\mathrm{N}$-acetamidoglucose units), 2.60 ( $\mathrm{CH}$, carbon 2 protons of $\mathrm{N}$-unsubstituted glucosamine units), 3.0-4.1 (CH, carbon 3, 4,5 , and 6 of glucosamine units; $\mathrm{CH}_{2}$, methylene protons of glycol residues of glycol chitosan polymer chains), 4.35 ( $\mathrm{CH}$, carbon 1 of glycol chitosan polymer chains). In contrast with the glycol chitosan, in the spectra of CHGC (Figure 2B), the new signals at $1.25-1.27 \mathrm{ppm}$ belonged to the methene hydrogen of CHS group. ${ }^{16}$ These results indicated that the CHGC conjugate was successfully synthesized. The colloidal titration method, which is based on the reaction between positively and negatively charged polyelectrolytes, was adopted to determine the amount of cholesterol group conjugated onto the glycol chitosan backbone. The DS was 5.9 cholesterol groups per 100 sugar residues of glycol chitosan. The CHGC conjugate formed self-assembled micelles in aqueous media. The intensity mean diameter of the CHGC micelles was determined to be $214 \mathrm{~nm}$ using DLS, and its zeta potential was $13.11 \mathrm{mV}$ (Table 1). Zeta potential is an important parameter related to the stability of micellar systems. A relatively high surface charge may present a repelling force between the particles and then increase the stability of the solution. ${ }^{37}$ In this study, the positive zeta potential of the micelles was attributed to the presence of ionized amino groups on the glycol chitosan backbone.

\section{Characterization of NCHGC micelles}

The CHGC copolymer was conjugated with NLS in the presence of SMCC, which is a widely used cross-linker for the conjugation of compounds with amino and thiol groups, 

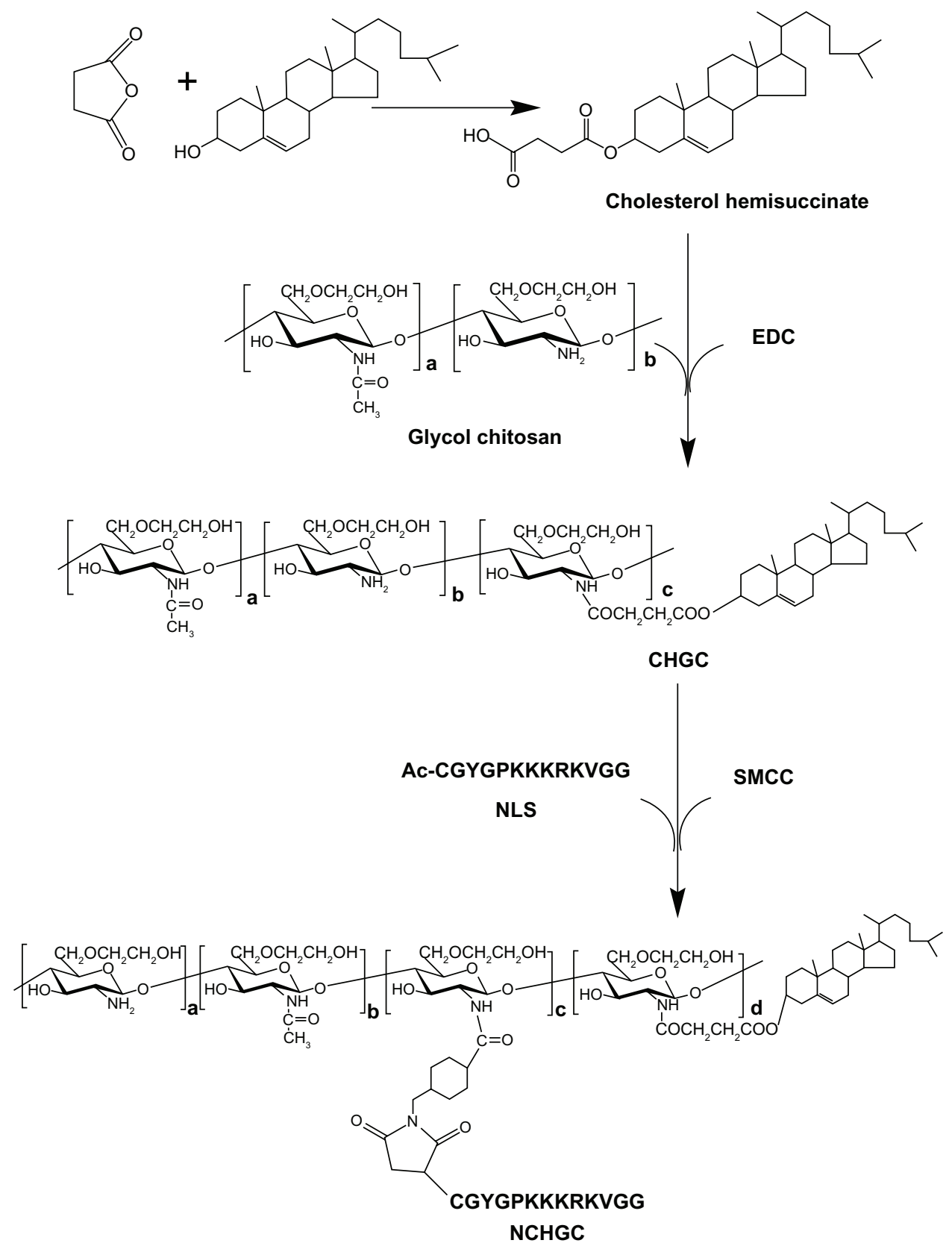

Figure I Synthetic scheme of NCHGC.

Abbreviations: EDC, I-ethyl-3-(3-dimethylaminopropyl)-carbodiimide hydrochloride; CHGC, cholesterol-modified glycol chitosan; SMCC, N-succinimidyl 4-(maleimidomethyl) cyclohexane carboxylate; NCHGC, nuclear localization signal-conjugated cholesterol-modified glycol chitosan; NLS, nuclear localization signal.

especially in protein-/peptide-related conjugation..$^{38,39}$ The Ac-CGYGPKKKRKVGG peptide is a SV40 T-antigen homolog, which is capable of inducing nuclear transport. Thus, we conjugated this NLS peptide to CHGC micelles so that NCHGC micelles might be used as drug carriers for active targeting of the cellular nucleus. Figure 1 shows NCHGC conjugate synthesis as a scheme. The ${ }^{1} \mathrm{H}-\mathrm{NMR}$ spectra of the NCHGC copolymer indicated that the NLS moiety was successfully conjugated to $\mathrm{CHGC}$, as the peaks of NLS appeared at 7.03, 6.76, and 0.88 ppm (Figure 2D).
The DS determined by Ellman's assay was 3.8 NLS groups per 100 sugar residues of NCHGC conjugate. The intensity particle size of NCHGC micelles analyzed by DLS was $205 \mathrm{~nm}$, and its zeta potential was $11.87 \mathrm{mV}$, which is similar to that of the CHGC micelles.

\section{Micellization behavior of NCHGC}

The micellization behavior of the NCHGC micelles in aqueous media was monitored by fluorescence spectroscopy in the presence of pyrene as a fluorescence probe. Pyrene 

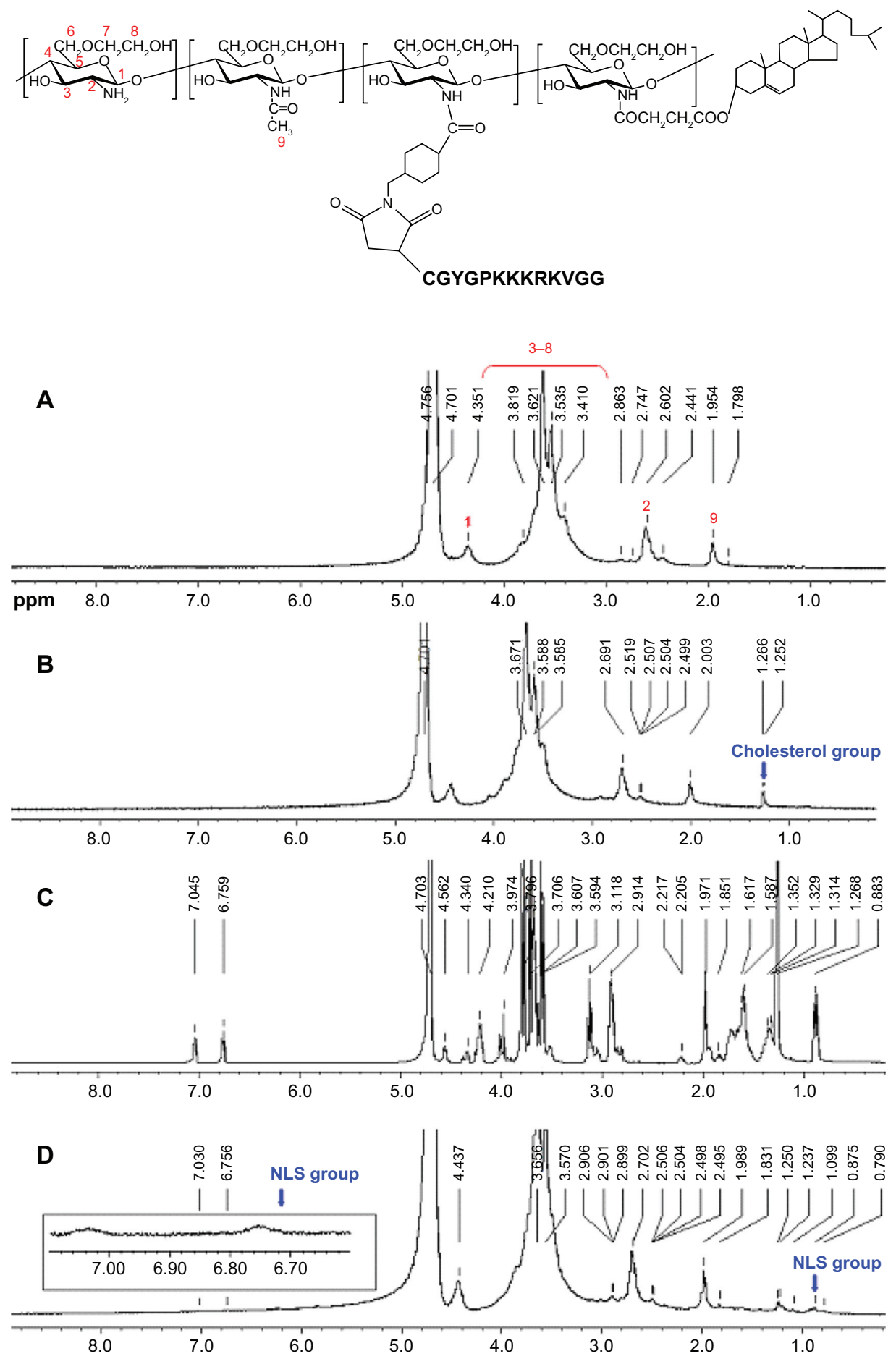

Figure 2 Proton nuclear magnetic resonance spectra of (A) glycol chitosan, (B) cholesterol-modified glycol chitosan (CHGC), (C) nuclear localization signal (NLS) (AcCGYGPKKKRKVGG), and (D) NLS-conjugated cholesterol-modified glycol chitosan (NCHGC).

molecules are reported to preferentially locate inside or close to the hydrophobic core of micelles. ${ }^{40}$ As shown in Figure 3, the value of the $I_{338} / I_{333}$ ratio changed with increasing concentrations of the polymers, indicating the transfer of pyrene molecules from a water environment to a hydrophobic micellar microdomain. The CAC of the NCHGC micelles was $0.0209 \mathrm{mg} / \mathrm{mL}$. A low CAC is desirable so that stable micelles can be formed during the dilution of drug-delivery systems by body fluid.

\section{Preparation and characterization of DOX-loaded micelles}

The DCHGC and DNCHGC micelles were prepared by an emulsion/solvent evaporation method. The DNCHGC micelles 
Table I Physicochemical properties of drug-free and drug-loaded micelles

\begin{tabular}{lllllll}
\hline Sample & DOX/carrier $^{\mathbf{a}}$ & Size $(\mathbf{n m})^{\mathbf{b}}$ & PI & Zeta potential $(\mathbf{m V})$ & LC $(\%)$ & EE $(\%)$ \\
\hline CHGC & - & $214 \pm 25.6$ & $0.226 \pm 0.073$ & $13.11 \pm 0.72$ & - & - \\
NCHGC & - & $205 \pm 18.7$ & $0.233 \pm 0.068$ & $11.87 \pm 0.89$ & - & - \\
DCHGC & $1.5 / 10$ & $246 \pm 22.3$ & $0.219 \pm 0.076$ & $11.93 \pm 0.55$ & $11.5 \pm 0.85$ & $86.7 \pm 6.41$ \\
DNCHGC & $1.5 / 10$ & $248 \pm 20.1$ & $0.241 \pm 0.083$ & $19.05 \pm 1.22$ & $10.1 \pm 0.79$ & $75.0 \pm 6.52$ \\
\hline
\end{tabular}

Notes: ${ }^{\text {TT }}$ The ratio of DOX to carrier, based on feed amount $(\mathrm{mg} / \mathrm{mg})$; ${ }^{b}$ measured by dynamic light scattering and described in terms of intensity distributions. The results represent the means \pm standard deviation $(n=3)$.

Abbreviations: DOX, doxorubicin; PI, polydispersity index; LC, loading content; EE, entrapment efficiency; CHGC, cholesterol-modified glycol chitosan; DCHGC, doxorubicin-loaded cholesterol-modified glycol chitosan; NCHGC, nuclear localization signal-conjugated cholesterol-modified glycol chitosan; DNCHGC, doxorubicinloaded nuclear localization signal-conjugated cholesterol-modified glycol chitosan.

that formed in aqueous medium are shown in Figure 4. TEA was used as a base to deprotonate the glycosidic amino group of DOX. As the molar ratio of TEA/DOX was fixed at 3.0, the DOX base could easily partition into the chloroform phase. The physicochemical properties of micelles, such as morphology, size, and zeta potential, are important parameters that influence cellular uptake. The physicochemical properties of the drug-loaded micelles in this study are summarized in Table 1. It was found that the mean diameters of the drug-loaded micelles were larger than those of the drug-free micelles, which implies that the DOX molecules were entrapped into the hydrophobic inner cores, which lead to the increase in the particle size of the micelles. The LC of DCHGC and DNCHGC micelles was $11.5 \%$ and $10.1 \%$, respectively. Moreover, the EE of DCHGC and DNCHGC micelles was $>75 \%$, indicating the good loading effect of the polymeric micelles. The zeta potentials of DCHGC and DNCHGC micelles were 11.93 and $19.05 \mathrm{mV}$, respectively. It is known that the surface of a cell membrane is negatively charged. Therefore, positively charged micelles can easily interact with cells and be effectively internalized. In a stability test, the particle size of the DOX-loaded micelles did not change in PBS (pH 7.4) for 7 days at room temperature (data not shown).

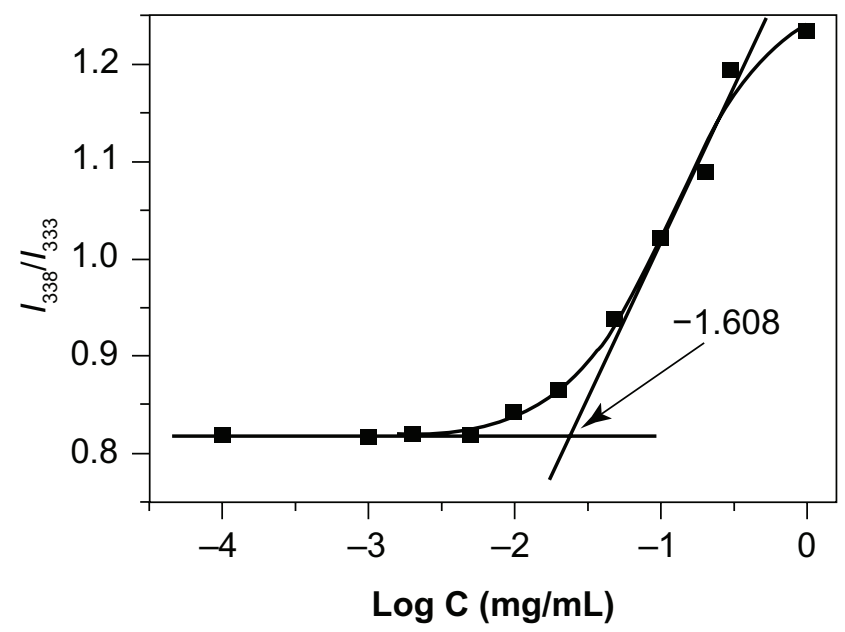

Figure 3 Plots of the intensity ratio $I_{338} I_{333}$ vs $\log C$ for nuclear localization signalconjugated cholesterol-modified glycol chitosan with various compositions.
As shown in Figure 5, transmission electron microscopy (TEM) observation demonstrated that the shape of the DNCHGC self-aggregates was roughly spherical. The mean diameter of the DNCHGC micelles was about $200 \mathrm{~nm}$, which is slightly smaller than the intensity particle size of $248 \mathrm{~nm}$ found when analyzed by DLS. This was mainly attributed to the process involved in the preparation of the sample. Further, TEM depicted the size of the sample in the dried state, whereas DLS determined the size in the hydrated state. Therefore, the size according to DLS analysis was a hydrodynamic diameter and was larger than the size measured by TEM because of the solvent effect. Similar results have been reported by other scientists. ${ }^{41,42}$

\section{In vitro DOX release}

In vitro release behavior studies of DOX were carried out in PBS containing $10 \% \mathrm{FBS}$ at $37^{\circ} \mathrm{C}$. During these experiments, the sink conditions were maintained by replacing the entire dialysis media. As shown in Figure 6, the total amounts of DOX released from the DCHGC and DNCHGC micelles over 72 hours were $59.4 \%$ and $58.1 \%$, respectively. It was also found that the DCHGC and DNCHGC micelles showed similar release behavior. In addition, DOX was released from the DOX-loaded micelles in a biphasic manner, which displayed an initial rapid release in 6 hours and a step of sustained release for up to 72 hours. This result indicates that the micelle-delivered DOX might stay in the micelle cores for a long time when the micelles are injected into plasma. Thus, sustained-release drug systems may offer the advantages of prolonged drug activity.

\section{Flow cytometry}

To determine the cellular uptake of free DOX, DCHGC, and DNCHGC micelles by HeLa cells after 2 hours incubation, flow cytometry was performed. As shown in Figure 7, the highest amount of cell-associated fluorescence was observed in HeLa cells that had been incubated with DNCHGC micelles. Moreover, HeLa cells incubated with DCHGC 


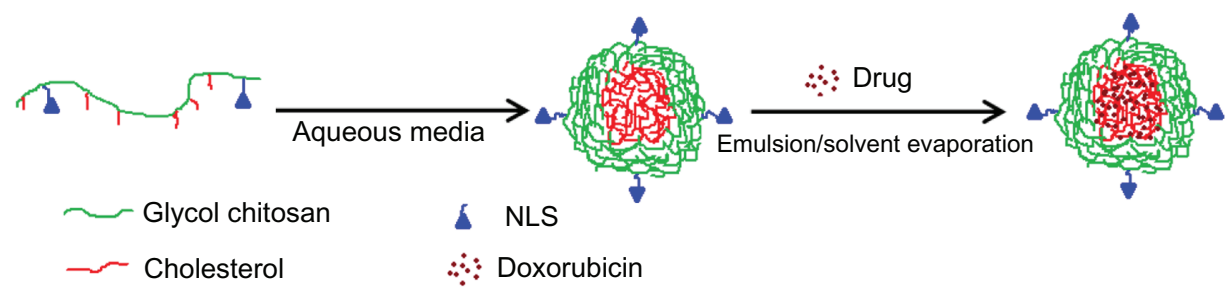

Figure 4 Schematic illustration of the preparation procedure of doxorubicin-loaded nuclear localization signal-conjugated cholesterol-modified glycol chitosan micelles by an emulsion/solvent evaporation method.

Abbreviation: NLS, nuclear localization signal (Ac-CGYGPKKKRKVGG).

micelles emitted higher fluorescent intensity than the cells treated with free DOX. Based on the release characteristic of DOX-loaded micelles, nearly $60 \%$ of the DOX remained in the micelles at 2 hours. About $40 \%$ of the DOX released from DOX-loaded micelles could contact with the membrane of HeLa cells. Therefore, DOX molecules from the micelles diffused into the cells more slowly than free DOX in solution. It can be concluded, therefore, that the micelles enhanced cellular internalization, and that free DOX and DOX-loaded micelles were in a different way into the cells.

\section{Confocal microscope study}

The intracellular fate of the micelles was exhaustively studied. ${ }^{43}$ The cellular uptake of micelles is an endocytic process. The nanoparticulate drug carriers were internalized as intact particles, whereas the free drug diffused throughout the cytoplasm and nucleus. It has been previously reported that in the presence of NLS peptides, carriers could efficiently enter the cellular nucleus. ${ }^{44-46}$ To directly monitor the cellular localization of DOX, three kinds of DOX formulations were incubated with HeLa cells for 2 hours and imaged with

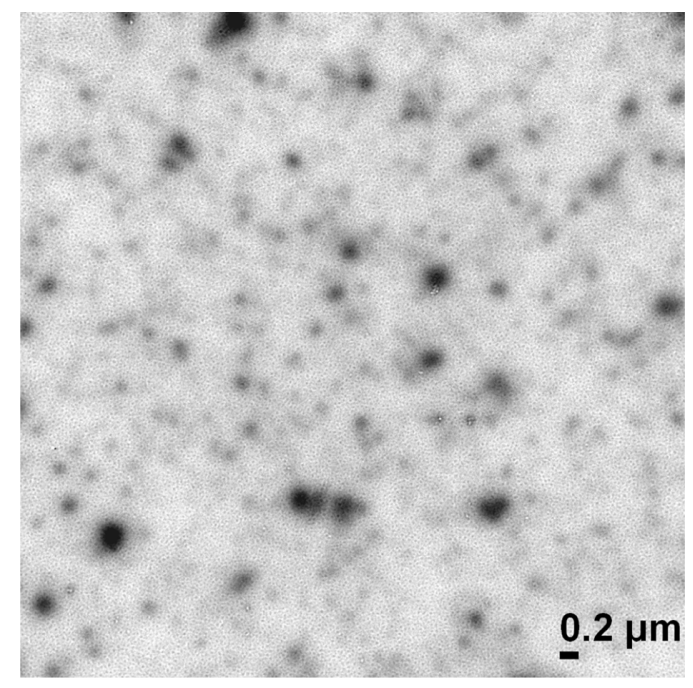

Figure 5 Transmission electron microscopy image of doxorubicin-loaded nuclear localization signal-conjugated cholesterol-modified glycol chitosan micelles $(\times 20,000)$. confocal laser scanning microscopy. As presented in Figure 8 most of the visible red fluorescence of DCHGC and DNCHGC micelles was in the nucleus, which is stained in blue with Hoechst 33342. Weak DOX fluorescence in the cytoplasm was also observed. The cells treated with the DNCHGC micelles clearly exhibit a stronger red fluorescence than the cells incubated with DCHGC micelles and free DOX. This result was consistent with the flow cytometry analysis. As stated earlier, the DOX-loaded micelles showed sustained-release behavior in vitro, whereas the free DOX without micelle encapsulation could freely diffuse in the media. This result further indicates that polymeric micelles could improve the cellular uptake of DOX. More notably, glycol chitosan-based micelles of large particle size and high molecular weight are difficult to transport into the nucleus. Our aim was to utilize NLS peptides for crossing the nuclear membrane barrier. Among these DOX formulations, the strongest red fluorescence was emitted from the DNCHGC micelles in the nucleus. Therefore, we inferred that DNCHGC micelles could be effectively transfered into the nucleus from cytoplasm by NLS-mediated active transport. In addition, the DNCHGC

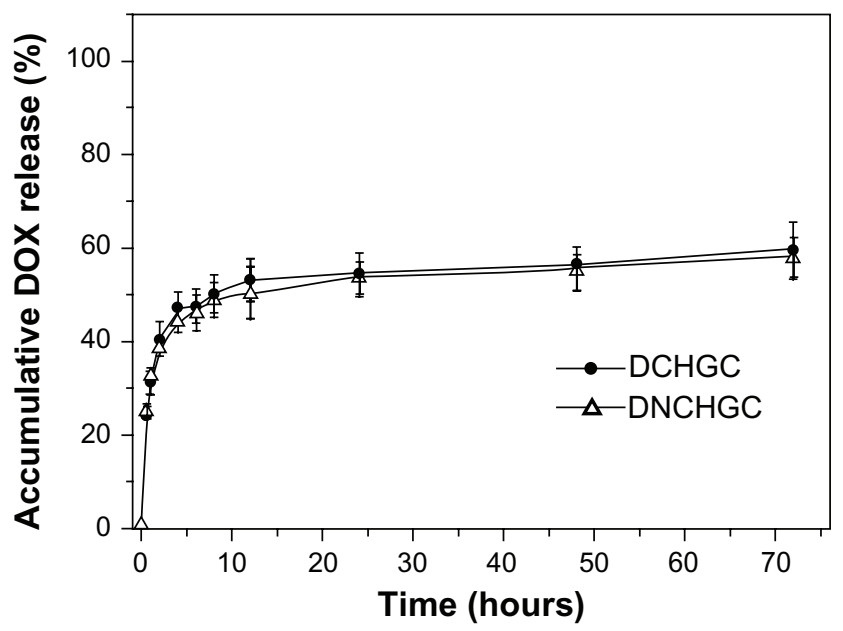

Figure 6 Release profiles of doxorubicin (DOX) from doxorubicin-loaded cholesterolmodified glycol chitosan (DCHGC) and doxorubicin-loaded nuclear localization signal-conjugated cholesterol-modified glycol chitosan (DNCHGC) micelles at $37^{\circ} \mathrm{C}$ in phosphate-buffered saline containing $10 \%$ fetal bovine serum at $\mathrm{pH}$ 7.4. 


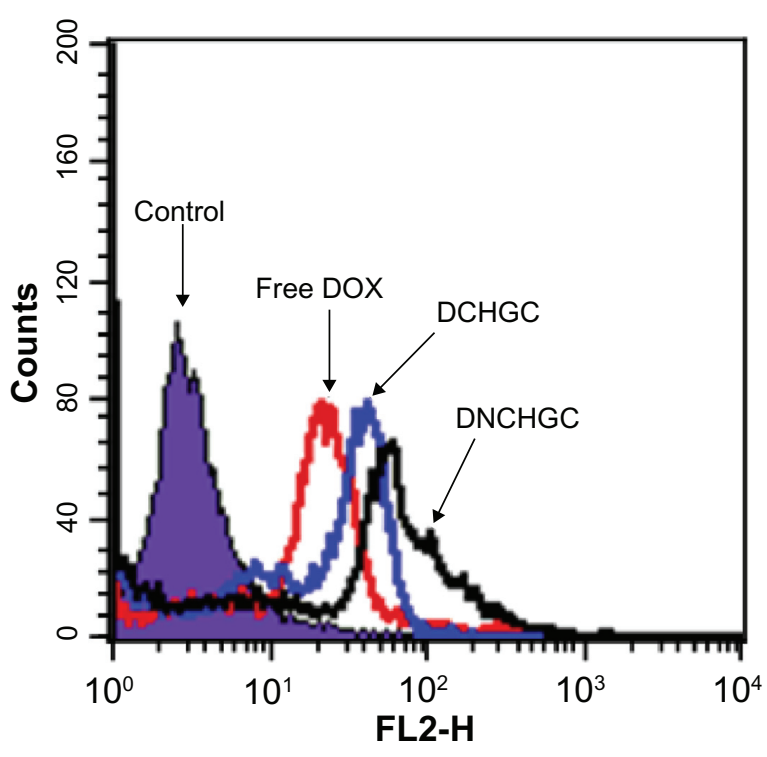

Figure 7 Flow cytometric analysis of HeLa cells incubated with free doxorubicin (DOX), doxorubicin-loaded cholesterol-modified glycol chitosan (DCHGC) and doxorubicin-loaded nuclear localization signal-conjugated cholesterol-modified glycol chitosan (DNCHGC) micelles for 2 hours.

micelles could also selectively bind to the NPC and allow a sustained release of DOX. Subsequently, free DOX could diffuse into the nucleus. ${ }^{22}$ These results suggest that DOXloaded micelles could improve the cytotoxicity of DOX.

\section{In vitro cytotoxicity}

The cytotoxicity of blank micelles, free DOX, and DOXloaded micelles against HeLa and HepG2 cells is shown

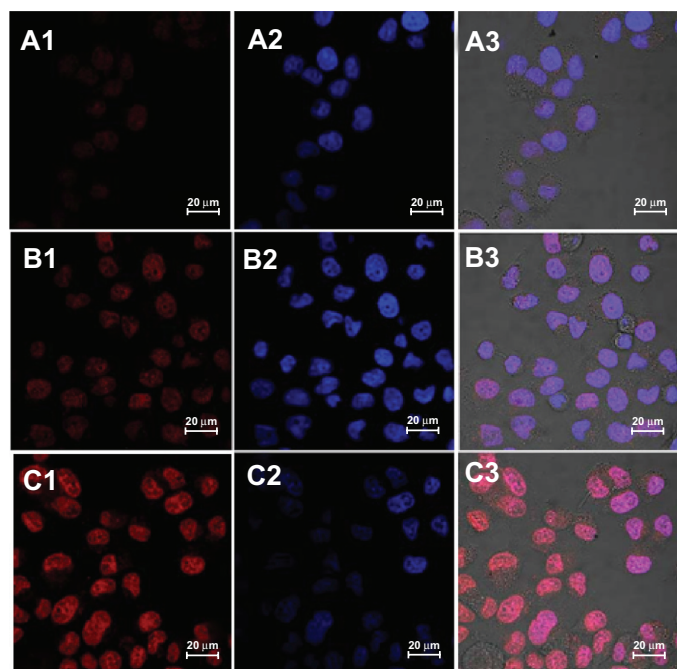

Figure 8 Confocal laser scanning microscopy images of HeLa cells after incubation with (A) free doxorubicin (DOX), (B) doxorubicin-loaded cholesterol-modified glycol chitosan micelles and (C) doxorubicin-loaded nuclear localization signalconjugated cholesterol-modified glycol chitosan micelles for 2 hours. The red DOX fluorescence was observed in $(\mathbf{A} \mathbf{I}-\mathbf{C I})$. The nuclei were stained with Hoechst 33342 in (A2-C2), and the merged photos were presented in (A3-C3).

in Figure 9. In the HepG2 cells, the $\mathrm{IC}_{50}$ values of blank CHGC and NCHGC micelles were 292.8 and $295.7 \mu \mathrm{g} /$ $\mathrm{mL}$, respectively (Figure 9A). Moreover, the $\mathrm{IC}_{50}$ values of CHGC and NCHGC micelles incubated with HeLa cells for 48 hours were 780.4 and $630.8 \mu \mathrm{g} / \mathrm{mL}$, respectively (Figure 9C). These results indicate that these conjugates showed low cytotoxicity against the test cells and did not interfere with the MTT test for micelle-entrapped DOX. As
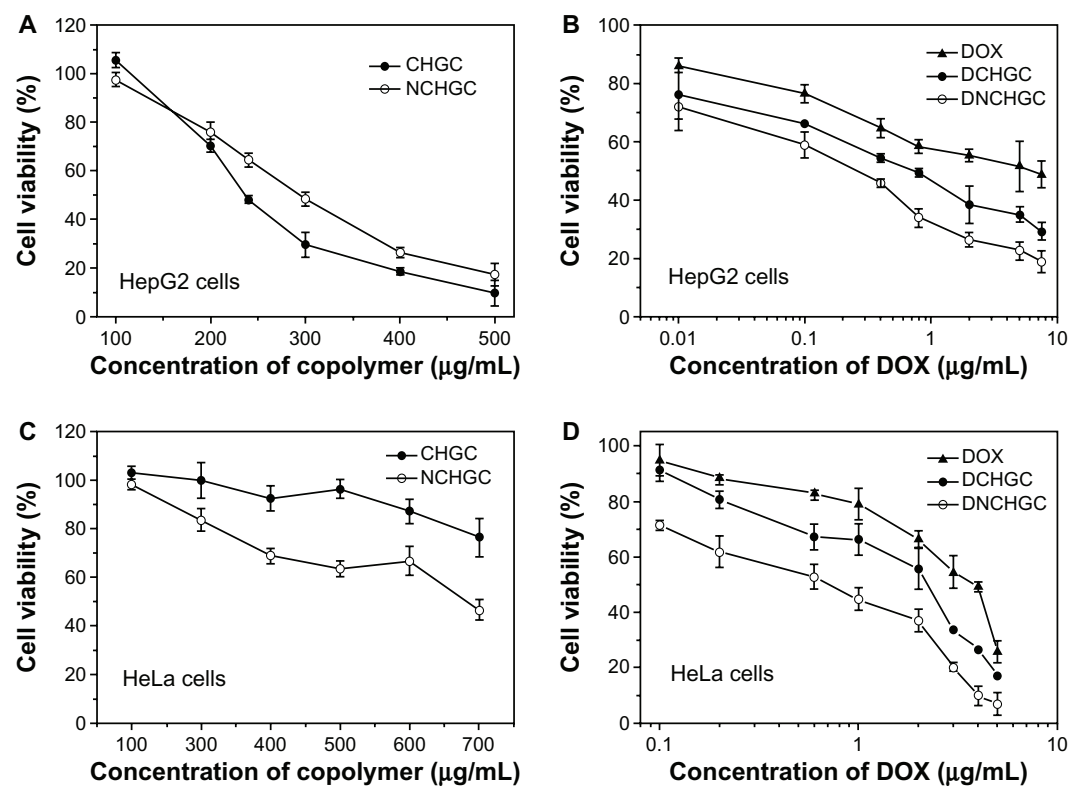

Figure 9 In vitro cytotoxicity of blank micelles and various doxorubicin (DOX) formulations against HepG2 and HeLa cells after 48 hours of incubation. (A) Cholesterolmodified glycol chitosan (CHGC) and nuclear localization signal-conjugated CHGC (NCHGC) micelles in HepG2 cells; (B) free DOX, doxorubicin-loaded CHGC (DCHGC) and doxorubicin-loaded nuclear localization signal-conjugated CHGC (DNCHGC) micelles in HepG2 cells; (C) CHGC and NCHGC micelles in HeLa cells; and (D) free DOX, DCHGC and DNCHGC micelles in HeLa cells. 
shown in Figure 9B, the DOX-loaded micelles exhibited much more potent cytotoxicity against HepG2 cells than free DOX. The $\mathrm{IC}_{50}$ values of free DOX, DCHGC, and DNCHGC micelles were 4.063, 0.591, and $0.171 \mu \mathrm{g} / \mathrm{mL}$, respectively. A similar result was observed in HeLa cells that the DNCHGC micelles exhibited the greatest cytotoxicity of the three DOX formulations (Figure 9D). The $\mathrm{IC}_{50}$ value of DNCHGC micelles $(0.461 \mu \mathrm{g} / \mathrm{mL})$ against HeLa cells was nearly 6.96 times $(P<0.01)$ lower than that of free DOX $(3.210$ $\mu \mathrm{g} / \mathrm{mL})$. Furthermore, the $\mathrm{IC}_{50}$ value of the DCHGC micelles was $1.413 \mu \mathrm{g} / \mathrm{mL}$, which is almost 2.27 times $(P<0.01)$ lower than that of the free DOX. As we know, the antitumor mechanism of DOX is the prevention of DNA replication and the inhibition of protein synthesis. DOX molecules in the nuclei were responsible for the cytotoxic effect of DOX formulations. Furthermore, from the in vitro release test, it was determined that DOX was released from the DCHGC and DNCHGC micelles over 48 hours in a sustained manner. Misra et al developed NLS-conjugated DOX-loaded poly (D,L-lactide-co-glycolide) nanoparticles, and these NLSconjugated nanoparticles exhibited a comparatively lower $\mathrm{IC}_{50}$ value than both the drug in solution and unconjugated nanoparticles in MCF-7 cells. ${ }^{22}$ As described earlier, in the present study, the DOX released from the DCHGC and DNCHGC micelles over 48 hours was almost $50 \%$ in vitro. Hence, the improved cytotoxicity is mainly due to the enhanced cellular uptake of DOX-loaded micelles and accumulation of DOX in the nuclear compartment. These results agree with flow cytometry and confocal microscopic observation. Therefore, the NCHGC micelles could be potential carriers for nucleus-targeting drug delivery.

\section{Conclusion}

In this study, novel NCHGC micelles were successfully synthesized and characterized. The antitumor drug DOX was physically loaded into NCHGC polymers to form coreshell structural micelles. The NCHGC micelles had a low CAC value and high drug-loading capacity. The DNCHGC micelles showed greater cellular uptake than the free DOX and DCHGC micelles in HeLa cells. Confocal observation showed that the DNCHGC micelles could effectively transport into the nucleus of HeLa cells and accelerate the accumulation of DOX. Compared with free DOX and DCHGC micelles, the cytotoxicities of the DNCHGC micelles against HeLa and HepG2 cells were significantly improved in vitro. Overall, these results demonstrate that the NCHGC micelle could be a promising drug carrier for nucleus-targeting delivery.

\section{Acknowledgments}

This work was financially supported by the National Nature Science Foundation of China (No 81001418), Scientific Research Fund of Jiangxi Health Department (No 210112028), Scientific Research Fund of Jiangxi Education Department (No GJJ11241), and Natural Science Foundation of Jiangxi Province, China (No 20114BAB215017). The authors are grateful for the helpful comments and careful corrections made by anonymous reviewers and editors.

\section{Disclosure}

The authors report no conflicts of interest in this work.

\section{References}

1. Sethuraman VA, Bae YH. TAT peptide-based micelle system for potential active targeting of anti-cancer agents to acidic solid tumors. J Control Release. 2007;118(2):216-224.

2. Seow WY, Xue JM, Yang YY. Targeted and intracellular delivery of paclitaxel using multi-functional polymeric micelles. Biomaterials. 2007;28(9):1730-1740.

3. Wang F, Wang YC, Dou S, Xiong MH, Sun TM, Wang J. Doxorubicintethered responsive gold nanoparticles facilitate intracellular drug delivery for overcoming multidrug resistance in cancer cells. ACS Nano. 2011;5(5):3679-3692.

4. Khonkarn R, Mankhetkorn S, Talelli M, Hennink WE, Okonogi S. Cytostatic effect of xanthone-loaded mPEG-b-p(HPMAm-Lac2) micelles towards doxorubicin sensitive and resistant cancer cells Colloid Surf B Biointerfaces. 2012;94:266-273.

5. Diao YY, Li HY, Fu YH, et al. Doxorubicin-loaded PEG-PCL copolymer micelles enhance cytotoxicity and intracellular accumulation of doxorubicin in adriamycin-resistant tumor cells. Int J Nanomedicine. 2011;6:1955-1962.

6. Matsumura Y, Maeda H. A new concept for macromolecular therapeutics in cancer chemotherapy: mechanism of tumoritropic accumulation of proteins and the antitumor agent smancs. Cancer Res. 1986; 46(12 Pt 1):6387-6392.

7. Torchilin V. Tumor delivery of macromolecular drugs based on the EPR effect. Adv Drug Deliver Rev. 2011;63(3):131-135.

8. Yuan XB, Li H, Yuan YB. Preparation of cholesterol-modified chitosan self-aggregated nanoparticles for delivery of drugs to ocular surface. Carbohydr Polym. 2006;65(3):337-345.

9. Uchegbu IF, Sadiq L, Pardakhty A, et al. Gene transfer with three amphiphilic glycol chitosans - the degree of polymerisation is the main controller of transfection efficiency. J Drug Target. 2004;12(8): $527-539$.

10. Kumar MN, Muzzarelli RA, Muzzarelli C, Sashiwa H, Domb AJ. Chitosan chemistry and pharmaceutical perspectives. Chem Rev. 2004; 104(12):6017-6084.

11. Kumar S, Dutta J, Dutta PK. Preparation and characterization of N-heterocyclic chitosan derivative based gels for biomedical applications. Int J Biol Macromol. 2009;45(4):330-337.

12. Lee SJ, Park K, Oh YK, et al. Tumor specificity and therapeutic efficacy of photosensitizer-encapsulated glycol chitosan-based nanoparticles in tumor-bearing mice. Biomaterials. 2009;30(15):2929-2939.

13. Hyung Park J, Kwon S, Lee M, et al. Self-assembled nanoparticles based on glycol chitosan bearing hydrophobic moieties as carriers for doxorubicin: in vivo biodistribution and anti-tumor activity. Biomaterials. 2006;27(1):119-126.

14. Kim JH, Kim YS, Kim S, et al. Hydrophobically modified glycol chitosan nanoparticles as carriers for paclitaxel. J Control Release. 2006;111(1-2):228-234. 
15. Yoo HS, Lee JE, Chung H, Kwon IC, Jeong SY. Self-assembled nanoparticles containing hydrophobically modified glycol chitosan for gene delivery. J Control Release. 2005;103(1):235-243.

16. Yu JM, Li YJ, Qiu LY, Jin Y. Self-aggregated nanoparticles of cholesterol-modified glycol chitosan conjugate: preparation, characterization, and preliminary assessment as a new drug delivery carrier. Eur Polym J. 2008;44(3):555-565.

17. Yu JM, Li YJ, Qiu LY, Jin Y. Polymeric nanoparticles of cholesterolmodified glycol chitosan for doxorubicin delivery: preparation and in-vitro and in-vivo characterization. J Pharm Pharmacol. 2009;61(6): 713-719.

18. Kalaria DR, Sharma G, Beniwal V, Ravi Kumar MN. Design of biodegradable nanoparticles for oral delivery of doxorubicin: in vivo pharmacokinetics and toxicity studies in rats. Pharm Res. 2009;26(3): 492-501.

19. Niu J, Su Z, Xiao Y, et al. Octreotide-modified and pH-triggering polymeric micelles loaded with doxorubicin for tumor targeting delivery. Eur J Pharm Sci. 2012;45(1-2):216-226.

20. Xiong XB, Huang Y, Lu WL, et al. Enhanced intracellular delivery and improved antitumor efficacy of doxorubicin by sterically stabilized liposomes modified with a synthetic RGD mimetic. J Control Release. 2005;107(2):262-275.

21. Kanazawa T, Takashima Y, Murakoshi M, Nakai Y, Okada H. Enhancement of gene transfection into human dendritic cells using cationic PLGA nanospheres with a synthesized nuclear localization signal. Int J Pharm. 2009;379(1):187-195.

22. Misra R, Sahoo SK. Intracellular trafficking of nuclear localization signal conjugated nanoparticles for cancer therapy. Eur J Pharm Sci. 2010;39(1-3):152-163.

23. Austin LA, Kang B, Yen CW, El-Sayed MA. Nuclear targeted silver nanospheres perturb the cancer cell cycle differently than those of nanogold. Bioconjug Chem. 2011;22(11):2324-2331.

24. Kang B, Mackey MA, El-Sayed MA. Nuclear targeting of gold nanoparticles in cancer cells induces DNA damage, causing cytokinesis arrest and apoptosis. J Am Chem Soc. 2010;132(5):1517-1519.

25. Sui MH, Liu WW, Shen Y. Nuclear drug delivery for cancer chemotherapy. J Control Release. 2011;155(2):227-236.

26. Lange A, Mills RE, Lange CJ, Stewart M, Devine SE, Corbett AH. Classical nuclear localization signals: definition, function, and interaction with importin alpha. J Biol Chem. 2007;282(8):5101-5105.

27. Xia WS, Liu P, Liu J. Advance in chitosan hydrolysis by non-specific cellulases. Bioresour Technol. 2008;99(15):6751-6762.

28. Hu FQ, Ren GF, Yuan H, Du YZ, Zeng S. Shell cross-linked stearic acid grafted chitosan oligosaccharide self-aggregated micelles for controlled release of paclitaxel. Colloid Surf B Biointerfaces. 2006; 50(2):97-103.

29. Kwon S, Park JH, Chung H, et al. Physicochemical characteristics of self-assembled nanoparticles based on glycol chitosan bearing $5 \beta$-cholanic acid. Langmuir. 2003;19(24):10188-10193.

30. Du YZ, Wang L, Yuan H, et al. Preparation and characteristics of linoleic acid-grafted chitosan oligosaccharide micelles as a carrier for doxorubicin. Colloid Surf B Biointerfaces. 2009;69(2):257-263.
31. Park Y, Hong HY, Moon HJ, et al. A new atherosclerotic lesion probe based on hydrophobically modified chitosan nanoparticles functionalized by the atherosclerotic plaque targeted peptides. $J$ Control Release. 2008;128(3):217-223.

32. Qiu LY, Yan MQ. Constructing doxorubicin-loaded polymeric micelles through amphiphilic graft polyphosphazenes containing ethyl tryptophan and PEG segments. Acta Biomater. 2009;5(6):2132-2141.

33. Du YZ, Weng Q, Yuan H, et al. Synthesis and antitumor activity of stearate-g-dextran micelles for intracellular doxorubicin delivery. ACS Nano. 2010;4(11):6894-6902.

34. Qiu LY, Wu XL, Jin Y. Doxorubicin-loaded polymeric micelles based on amphiphilic polyphosphazenes with poly(N-isopropylacrylamide-coN,N-dimethylacrylamide) and ethyl glycinate as side groups: synthesis, preparation and in vitro evaluation. Pharm Res. 2009;26(4):946-957.

35. Sakai-Kato K, Ishikura K, Oshima Y, et al. Evaluation of intracellular trafficking and clearance from HeLa cells of doxorubicin-bound block copolymers. Int J Pharm. 2012;423(2):401-409.

36. Qiu L, Zhang L, Zheng C, et al. Improving physicochemical properties and doxorubicin cytotoxicity of novel polymeric micelles by poly (E-caprolactone) segments. J Pharm Sci. 2011;100(6):2430-2442.

37. Liang N, Sun S, Li X, et al. $\alpha$-Tocopherol succinate-modified chitosan as a micellar delivery system for paclitaxel: preparation, characterization and in vitro/in vivo evaluations. Int J Pharm. 2012;423(2):480-488.

38. Liang JF, Yang VC. Synthesis of doxorubicin-peptide conjugate with multidrug resistant tumor cell killing activity. Bioorg Med Chem Lett. 2005; 15(22):5071-5075.

39. Aroui S, Ram N, Appaix F, et al. Maurocalcine as a non toxic drug carrier overcomes doxorubicin resistance in the cancer cell line MDA-MB 231. Pharm Res. 2009;26(4):836-845.

40. Zhang J, Ma PX. Polymeric core-shell assemblies mediated by hostguest interactions: versatile nanocarriers for drug delivery. Angew Chem Int Ed Engl. 2009;48(5):964-968.

41. Sajomsang W, Gonil P, Ruktanonchai UR, et al. Self-aggregates formation and mucoadhesive property of water-soluble $\beta$-cyclodextrin grafted with chitosan. Int J Biol Macromol. 2011;48(4):589-595.

42. Prabha S, Zhou WZ, Panyam J, Labhasetwar V. Size-dependency of nanoparticle-mediated gene transfection: studies with fractionated nanoparticles. Int J Pharm. 2002;244(1-2):105-115.

43. Torchilin VP. Fluorescence microscopy to follow the targeting of liposomes and micelles to cells and their intracellular fate. Adv Drug Deliver Rev. 2005;57(1):95-109.

44. Yoo HS, Jeong SY. Nuclear targeting of non-viral gene carriers using psoralen-nuclear localization signal (NLS) conjugates. Eur J Pharm Biopharm. 2007;66(1):28-33.

45. Wang HY, Li C, Yi WJ, et al. Targeted delivery in breast cancer cells via iodine: nuclear localization sequence conjugate. Bioconjugate Chem. 2011;22(8):1567-1575.

46. Hu Q, Wang J, Shen J, et al. Intracellular pathways and nuclear localization signal peptide-mediated gene transfection by cationic polymeric nanovectors. Biomaterials. 2012;33(4):1135-1145.
International Journal of Nanomedicine

\section{Publish your work in this journal}

The International Journal of Nanomedicine is an international, peerreviewed journal focusing on the application of nanotechnology in diagnostics, therapeutics, and drug delivery systems throughout the biomedical field. This journal is indexed on PubMed Central, MedLine, CAS, SciSearch ${ }^{\circledR}$, Current Contents ${ }^{\circledR} /$ Clinical Medicine,

\section{Dovepress}

Journal Citation Reports/Science Edition, EMBase, Scopus and the Elsevier Bibliographic databases. The manuscript management system is completely online and includes a very quick and fair peer-review system, which is all easy to use. Visit http://www.dovepress.com/ testimonials.php to read real quotes from published authors. 